\title{
New molecular tools for meningitis diagnostics in Ethiopia - a necessary step towards improving antimicrobial prescription
}

Guro K. Bårnes ${ }^{1,2}$, Esayas Kebede Gudina ${ }^{3}$, Melkamu Berhane ${ }^{3}$, Alemseged Abdissa ${ }^{3}$, Getnet Tesfaw³, Gemeda Abebe ${ }^{3,5}$, Siri Laura Feruglio ${ }^{1}$, Dominique A. Caugant ${ }^{1,2^{*}}$ (D) and Hannah Joan Jørgensen ${ }^{1,4}$

\begin{abstract}
Background: Meningitis remains a top cause of premature death and loss of disability-adjusted life years in lowincome countries. In resource-limited settings, proper laboratory diagnostics are often scarce and knowledge about national and local epidemiology is limited. Misdiagnosis, incorrect treatment and overuse of antibiotics are potential consequences, especially for viral meningitis.

Methods: A prospective study was conducted over three months in a teaching hospital in Ethiopia with limited laboratory resources. Cerebrospinal fluid (CSF) samples from patients with suspected meningitis were analysed using a multiplex PCR-based system (FilmArray, BioFire), in addition to basic routine testing with microscopy and culture. Clinical data, as well as information on treatment and outcome were collected.

Results: Two hundred and eighteen patients were included; 117 (54\%) neonates (0-29 days), 63 (29\%) paediatrics (1 month-15 years) and 38 (17\%) adults ( $\geq 16$ years). Of 218 CSF samples, 21 (10\%) were PCR positive; $4 \%$ in neonates, $14 \%$ in paediatrics and $18 \%$ in adults. Virus was detected in $57 \%$ of the PCR positive samples, bacteria in $33 \%$ and fungi in $10 \%$. All CSF samples that were PCR positive for a bacterial agent had a white cell count $\geq 75 \mathrm{cells} / \mathrm{mm}^{3}$ and/or turbid appearance. The majority (90\%) of patients received more than one antibiotic for treatment of the meningitis episode. There was no difference in the mean number of different antibiotics received or in the cumulative number of days with antibiotic treatment between patients with a microorganism detected in CSF and those without.

Conclusions: A rapid molecular diagnostic system was successfully implemented in an Ethiopian setting without previous experience of molecular diagnostics. Viral meningitis was diagnosed for the first time in routine clinical practice in Ethiopia, and viral agents were the most commonly detected microorganisms in CSF. This study illustrates the potential of rapid diagnostic tests for reducing antibiotic usage in suspected meningitis cases. However, the cost of consumables for the molecular diagnostic system used in this study limits its use in low-income countries.
\end{abstract}

Keywords: Cerebrospinal fluid, FilmArray, Multiplex PCR, Viral meningitis, Bacterial meningitis

\footnotetext{
* Correspondence: dominique.caugant@fhi.no

${ }^{1}$ Division for Infection Control and Environmental Health, Norwegian Institute of Public Health, Oslo, Norway

${ }^{2}$ Faculty of Medicine, University of Oslo, Oslo, Norway

Full list of author information is available at the end of the article
}

(c) The Author(s). 2018 Open Access This article is distributed under the terms of the Creative Commons Attribution 4.0 International License (http://creativecommons.org/licenses/by/4.0/), which permits unrestricted use, distribution, and reproduction in any medium, provided you give appropriate credit to the original author(s) and the source, provide a link to the Creative Commons license, and indicate if changes were made. The Creative Commons Public Domain Dedication waiver (http://creativecommons.org/publicdomain/zero/1.0/) applies to the data made available in this article, unless otherwise stated. 


\section{Background}

Meningitis remains a major cause of mortality and morbidity worldwide. In Ethiopia, it is an important cause of premature death and disability, being the 9th most common cause of years of life lost and loss of disability-adjusted life years [1]. In addition to the morbidity and mortality associated with meningitis, the disease also represents a huge burden on affected families and the health care system, especially in low-income countries. One case of meningococcal disease in sub-Saharan Africa has been estimated to cost the household 90 US dollar (USD), equal to $34 \%$ of the annual gross domestic product per capita, and an additional 154 USD if the disease leads to sequelae [2].

Early treatment is essential in the clinical management of meningitis. A delay in therapy negatively affects the prognosis for patients with both bacterial [3] and viral [4] meningitis/encephalitis. Empiric antimicrobial therapy is therefore initiated on suspicion, before laboratory results are available $[5,6]$. Traditionally, laboratory diagnostics of meningitis have included examination of cerebrospinal fluid (CSF) for white blood cells (WBC), measurement of glucose and protein levels, and Gram staining. These are rapid, low-cost analyses but do not have satisfactory specificity alone [7, 8]. When septicemia is additionally suspected, peripheral blood WBC count is usually obtained, but this analysis also suffers from a lack of sensitivity and specificity [9, 10]. Positive bacterial cultures from CSF can provide a definite diagnosis, but may take several days and quality requirements are high. Therefore, rapid molecular diagnostics have become routine in meningitis diagnostics in high-income countries.

In many low-income countries, poor laboratory services impede the quality of health care. Ethiopia is experiencing high gross domestic product growth, but the per capita income remains one of the lowest in the world [11]. Healthcare in Ethiopia is under-resourced, and laboratory services are weak. Inadequate and delayed microbiological services foster distrust in the value of laboratory testing among clinicians, and reduce their inclination to submit clinical samples for analyses. Ultimately, this compromises patient care, prevention of infectious diseases and antibiotic stewardship.

More often than not, in Ethiopia and other low-resource settings patients receive an inadequate diagnostic workup [12] and decisions to treat meningitis are based on clinical findings alone. This approach lacks accuracy $[13,14]$ and ultimately results in unjustified prescriptions of antibiotics [12]. Incorrect use of antimicrobials is a recognized challenge in Ethiopia, with delayed initiation, unindicated use, duplication of broad spectrum antimicrobials and unnecessarily long duration of treatment being the major problems [15]. Fortunately, in the last few years greater attention has been paid to antimicrobial resistance also in Ethiopia, where it is becoming an increasing concern [16].

Recently, a rapid molecular diagnostic system (FilmArray, BioFire; http://www.biomerieux-usa.com/clinical/biofire-film-array) was developed for multiplex testing for the most common causes of infectious syndromes. The system is closed and integrates nucleic acid extraction and nested PCR for detection of the pathogens included in the panels. It is easy to use, has a low risk of sample contamination, requires little hands-on time and limited prior training in molecular diagnostics. For each sample, a kit that contains all necessary reagents is used, and sample preparation takes only a couple of minutes. These features are advantageous in settings with limited experience and limited facilities for molecular analyses. The main limitation for use in such settings is the high cost of test-kits.

The aim of this study was to investigate which infectious agents were responsible for meningitis in a region of Ethiopia where treatment decisions are usually made without microbiological results. We demonstrated that an easy-to-use and reliable molecular diagnostic instrument could set the ground for improving patient management and reducing usage of antimicrobials in a resource-limited setting.

\section{Methods}

\section{Study design and population}

This prospective study was performed between March 20th and June 20th 2017, at Jimma University Specialized Hospital (JUSH), one of the oldest public hospitals in Ethiopia, established in 1937. It is the only teaching and referral hospital in the southwestern part of the country and serves a catchment population of 15-20 million. The hospital has 600 beds and 1621 staff members, providing services for 218,095 outpatients, 16,778 inpatients, 14,207 emergencies and 5,973 deliveries yearly (data from September 2016 to September 2017). Other public health services in the catchment area include five primary hospitals, one army hospital, 118 health centres and 482 health posts.

All patients with a clinical suspicion of meningitis and from whom a CSF sample was available for analyses were eligible for inclusion. The study was run as an integrated part of routine practice and inclusion was done by the treating physicians. A written consent was given by the participants, or by parents or guardians in the case of children or unconscious patients. As samples were taken as part of routine diagnostic practice, participation in the study required no additional interventions.

Before the start of the study, a general introduction of the project aims and procedures, to the new instrument and interpretation of results was given to all medical doctors at the pediatric and internal medicine department. A four-hour training on how to perform analysis 
with the FilmArray was given to four dedicated JUSH staff members at the bacteriology department. Results of the PCR analysis are generated automatically by the FilmArray software and are reported as either negative, or positive with a defined species. Two senior medical doctors (study investigators) associated with the pediatric and medical wards, respectively, acted as supervisors for other physicians on the wards during the project. They were responsible for follow-up, and supported treating physicians with clinical/treatment implications of the laboratory results. The first author, a medical doctor, was present at JUSH during the project period and followed up data collection. Sample analyses, reporting of results and clinical decisions about treatment were done by local JUSH staff.

\section{Clinical data, treatment, routine investigations and outcome}

Patient data were collected on a structured case record form. The treating physician collected the consent and clinical data at admission while microbiological analysis and results were documented by laboratory staff. Laboratory results were, according to routine at JUSH bacteriology laboratory, collected by the treating physician and the paper transcript of the laboratory result was kept in the patient chart. Results were also intended to be immediately reported to the treating physician by cellular phone. However, the latter additional reporting was not always complied with in practice. Data on treatment, routine investigations and outcome were collected by study investigators (all medical doctors), either at discharge or retrospectively within one month of discharge. Patients with none or incomplete data on treatment, routine investigations and outcome were excluded from the relevant statistical/data analyses, but included in analyses on clinical and laboratory data.

\section{Sample collection}

CSF samples were collected in sterile glass tubes for neonates and paediatrics and in sterile plastic tubes $(5 \mathrm{ml}$, Sartstedt, Germany) for adults. They were transported to the laboratory at ambient temperature within ten minutes and laboratory analyses were started within two hours, but usually within $30 \mathrm{~min}$. For patients with suspected concomitant septicemia, blood was collected in blood culture bottles using BD BACTEC Peds Plus (Becton Dickinson (BD), Franklin Lakes, NJ, USA) for paediatric patients and BD BACTEC Plus Aerobic medium (BD) for adult patients.

\section{Conventional laboratory analyses of CSF}

CSF samples were processed according to standard procedures at JUSH which include bacteriological culture, macroscopic assessment, protein and glucose measurements, manual WBC count with differential count and Gram staining. India ink staining and rapid cryptococcal antigen testing (CrAg, IMMY, OK, USA) were performed upon request. Gram stain was performed on the primary sample, usually without centrifugation due to lack of equipment and/or consumables. CSF glucose and protein measurements, were performed when reagents were available. CSF samples were cultured onto blood agar (BD) and/or chocolate agar (BD) for $72 \mathrm{~h}$ at $35^{\circ} \mathrm{C}$ in a $\mathrm{CO}_{2}$-enriched incubator (candle jar). Routine bacterial identification was based on colony morphology, Gram staining and standard biochemical reactions [17].

\section{Molecular diagnostic of CSF}

All CSF samples included in the study were analysed using the meningitis/encephalitis (ME) panel (bioMérieux, Marcy l'Etoile, France) on the FilmArray (bioMérieux,) multiplex PCR system. The closed system performs nucleic acid extraction, reverse transcription and multiplex nested PCR, automatically interprets end-point melting curve data and provides the result. The ME panel tests for genetic targets from Escherichia coli K1, Haemophilus influenzae, Listeria monocytogenes, Neisseria meningitidis, Streptococcus agalactiae, Streptococcus pneumoniae, cytomegalovirus, enterovirus, herpes simplex virus 1 (HSV-1), herpes simplex virus 2 (HSV-2), human herpesvirus 6 (HHV-6), human parechovirus, varicella zoster virus (VZV) and Cryptococcus neoformans/gattii. Analyses were performed according to the manufacturer's instructions [18]. A flow-chart of the sample analysis with the FilmArray is shown in Additional file 1: Figure S1.

\section{Other laboratory analysis}

CSF samples from patients with suspected tuberculous meningitis (clinical suspicion, HIV-positivity, or no improvement on conventional antibiotic treatment) were tested for Mycobacterium tuberculosis with the GeneXpert MTB/RIF assay (Dx System Version 4.0c, Cepheid Inc., CA, US) [19]. CSF was processed as recommended by the manufacturer: a 2:1 volume of the reagent supplied with the assay was added directly to the sample. The mixture was vortexed and incubated at room temperature for $15 \mathrm{~min}$. Two $\mathrm{mL}$ of the reagent-sample mix was then transferred to the Xpert cartridge and analysed.

Blood culture bottles were incubated in an automated blood culture incubator BACTEC FX40 system (BD) for a maximum of five days and flagged as either "negative" or "positive" for bacterial growth. Positive blood cultures were analysed using the FilmArray blood culture identification (BCID) panel (BioMerieux, France) [20], and cultured onto blood agar (BD), MacConkey agar (BD) and chocolate agar (BD), with the same routine bacterial identification as for CSF cultures. 
Other investigations, such as malaria testing using peripheral blood smear and May-Grünwald-Giemsa staining, rapid testing for HIV, haematological and biochemical analyses were performed according to routine practice at JUSH.

\section{Data management and statistical analysis}

All data were entered into a Microsoft Excel 2013 database. Statistical analyses were performed in Microsoft Excel 2013 or in GraphPad Prism 5. Two-sided tests were used and a $p$-value $<0.05$ was considered statistically significant.

\section{Results}

\section{Patient characteristics}

During the study period, CSF samples from 220 patients with suspected meningitis were analysed at the laboratory. Of these, 218 patients gave consent and were included in the study; $42 \%$ were female. The study population consisted of 117 (54\%) neonates (0-29 days), 63 (29\%) paediatrics (1 month-15 years) and 38 (17\%) adults ( $\geq 16$ years). Demographic data and the number of samples taken per age group is shown in Fig. 1.

\section{Laboratory results \\ Conventional laboratory investigations}

In 213 (97\%) of the 218 CSF samples, the macroscopic appearance was recorded. Glucose was measured in 42 (19\%) of the samples and a manual cell count was performed in 201 (92\%). In 49\% of these samples, no cells were detected, and $18 \%$ of the samples contained blood which prohibited the cell count. In the remaining 33\% of samples WBC were detected and a differential count was available for $91 \%$ of these. Gram stain was performed in 217 samples, but microorganisms were observed in only three (bacteria in two and fungal elements in one). In total, 213 (97\%) of the samples were cultured and only one sample was positive (Klebsiella pneumoniae) (Table 1). Hence, altogether only four (2\%) of the CSF samples included in the study were found to be positive for microorganisms using conventional methods (microscopy and/or culture).

\section{Molecular diagnostics}

In $21(10 \%)$ of the CSF samples, pathogens were detected by PCR using the FilmArray ME panel $(n=20)$ and BCID panel $(n=1)$. Using the ME panel, viral agents were detected in 57\% (HHV-6, HSV-1, enterovirus, HSV-2, varicella zoster virus), bacteria in 33\% (H. influenzae, S. pneumoniae, E. coli, N. meningitidis) and fungi in $10 \%$ (C. neoformans/gattii) (Fig. 2). Only one CSF sample was culture positive, but this sample was negative by FilmArray using the ME-panel. The culture was identified as $K$. pneumoniae, which is not included in the ME panel. The CSF sample was therefore analysed by FilmArray using the BCID panel, which confirmed positivity. A blood culture sample from the same patient was also $K$. pneumoniae-positive by the BCID panel.

The proportion of positive CSF samples increased with age with $4 \%$ in neonates, $14 \%$ in paediatrics and $18 \%$ in adults (Fig. 1). Table 2 shows the results of the CSF analyses and patient outcome for samples where a pathogen was detected.

Thirty two (15\%) participants had blood cultures taken. Overall $12(38 \%)$ of these were positive including 1 of 2 samples from adults, 5 of 21 samples from paediatrics and 6 of 9 samples from neonates. Two patients were positive for microorganisms in both CSF and blood culture. One patient had $K$. pneumoniae detected in both samples, CSF by culture and blood by FilmArray, while the other had HSV-1 detected in CSF and Enterococcus spp. detected in blood, both by FilmArray. For positive blood cultures, routine culture confirmed bacterial isolates in all samples, although in one individual, only $K$. pneumoniae was identified by culture, while the BCID panel in addition to identifying $K$. pneumoniae

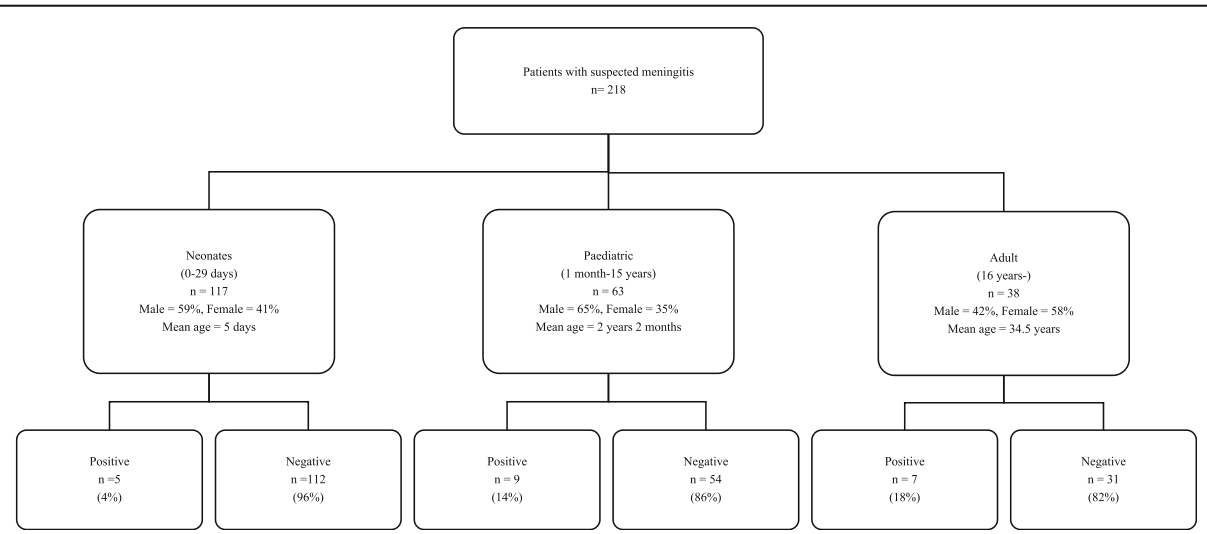

Fig. 1 Study participants and results from analyses of cerebrospinal fluid (CSF) samples using FilmArray and culture with respect to detection of a microorganism (positive) or not (negative). Flow chart of study participants, demographics and CSF samples 


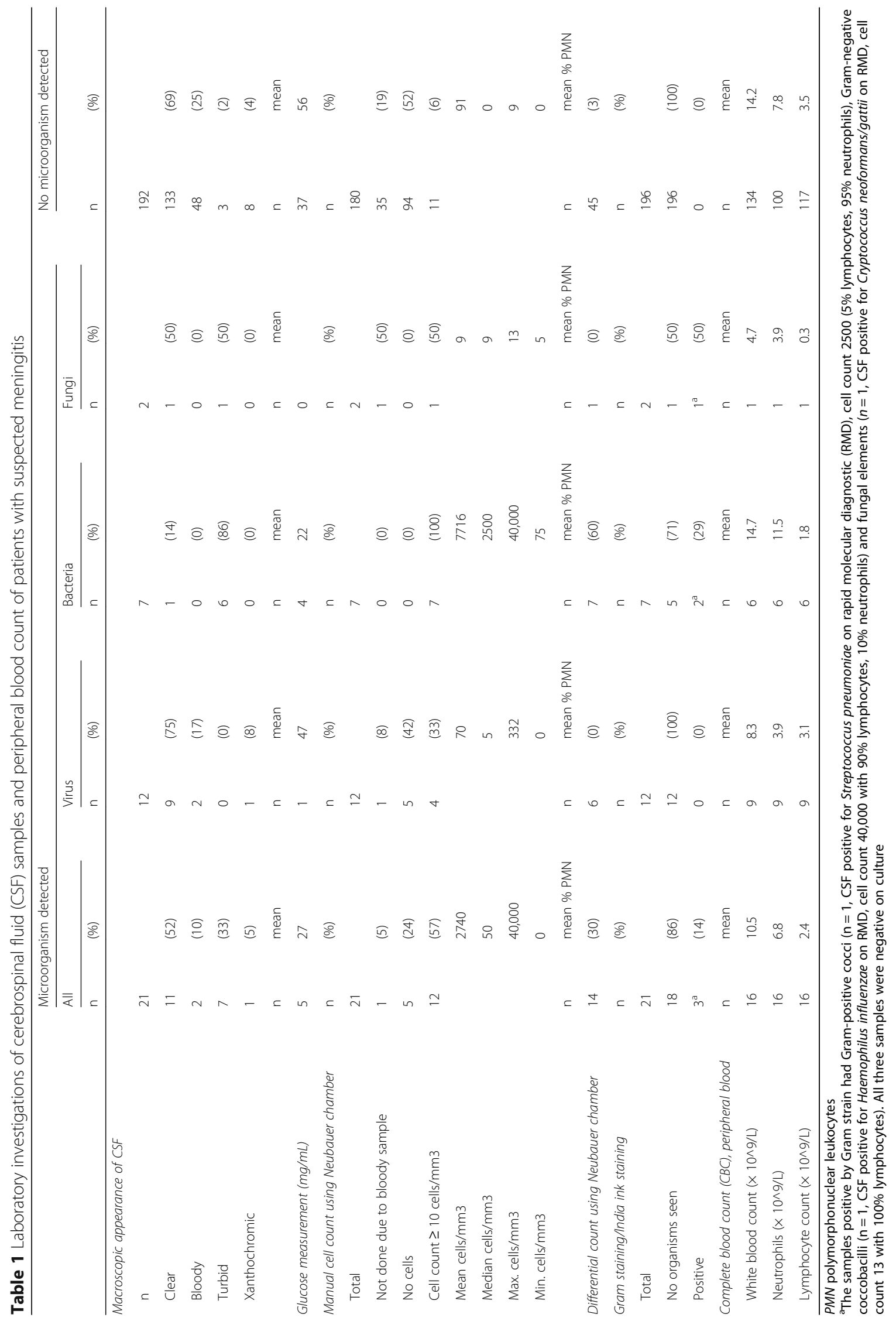




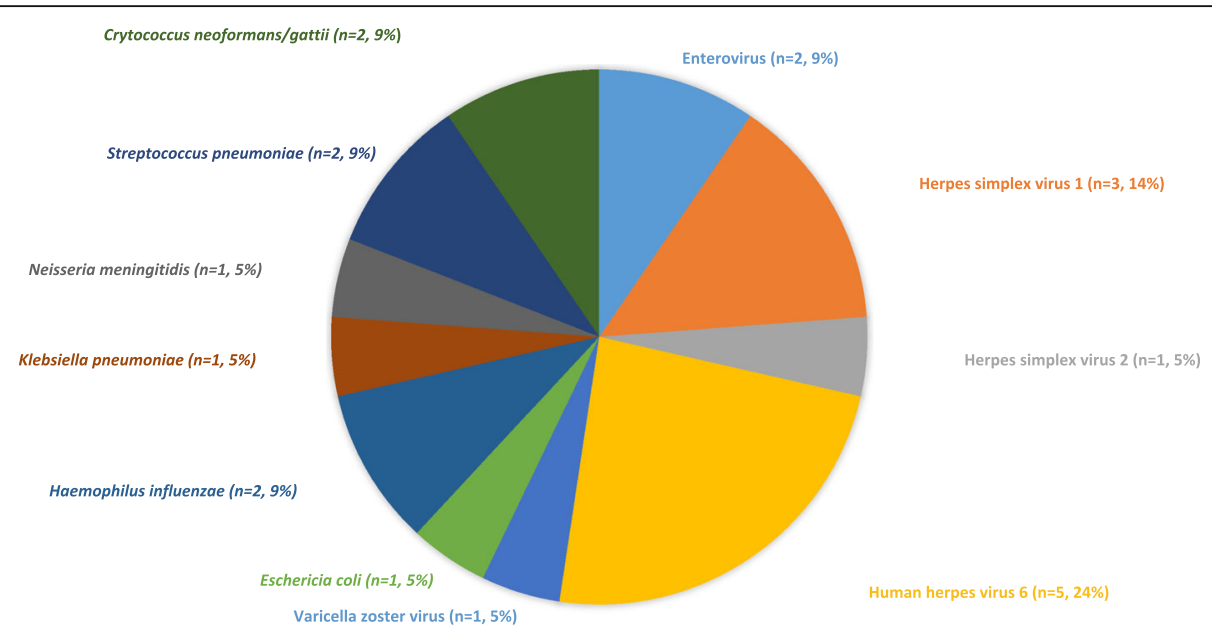

Fig. 2 Etiological agents detected in cerebrospinal fluid (CSF) samples from patients with suspected meningitis using FilmArray and culture. Pie chart of etiological agents detected in CSF samples from patients with suspected meningitis

also detected Enterobacter cloacae complex. Three positive blood cultures, in which no agent was detected by FilmArray, were positive by routine culture for Staphylococcus spp, Acinetobacter spp. and Salmonella spp., respectively.

CSF samples from 20 patients, 9 paediatrics and 11 adults, were analysed for $M$. tuberculosis using the GeneXpert system, and all were negative. Three samples were tested using the cryptococcal antigen test, and two of these were positive. These two samples were also positive for $C$. neoformans/gattii by FilmArray (ME panel).

\section{Other diagnostic tests}

Data on testing for malaria (tested or not tested) were available for 178 patients. In total, 43 (24\%) were tested, all using peripheral blood smear. Only one patient was positive, with a mixed infection of Plasmodium falciparum and Plasmodium vivax.

HIV status was known for 44 (19\%) patients and 12 (27\%) of these were HIV positive. Both patients with meningitis caused by $C$. neoformans/gattii had advanced stages of HIV infection.

\section{Laboratory results in samples where microorganisms were detected versus not detected}

A comparison was made of laboratory results from analyses of CSF samples where microorganisms were detected and samples where no microorganism was detected (Table 1). There was a significant difference (OR, 28.2; 95\%CI 6.4-124.6; $p<0.0001$ ) for detecting a pathogen in turbid versus clear samples. In all turbid samples where a pathogen was detected, this was either bacteria or fungi. However, not all samples where bacteria or fungi were detected were turbid.

Glucose measurements were generally lower in samples with bacteria (mean $22 \mathrm{mg} / \mathrm{dL}$ ) compared to samples where virus (mean $47 \mathrm{mg} / \mathrm{dL}$ ) or no microorganism (mean $56 \mathrm{mg} / \mathrm{dL}$ ) were detected, but the number of available measurements was low (Table 1).

For all samples where microorganisms were detected by Gram stain, a pathogen was also detected by FilmArray or culturing. All CSF samples in which bacteria were detected by PCR or culture (Table 3) had a cell count $\geq 75$ cells $/ \mathrm{mm}^{3}$ and/or turbid appearance. In this group of samples ( $\geq 75$ cells $/ \mathrm{mm}^{3}$ and/or turbid appearance) a bacterial agent was detected in $44 \%$.

The majority of patients had other laboratory investigations performed in addition to CSF analysis. The most common laboratory test was complete blood count in peripheral blood, but no differences were seen in mean WBC count between patients with positive and negative CSF samples (Table 1). However, patients with confirmed bacterial meningitis had a higher mean proportion of polymorphonuclear cells (PMN) in their peripheral blood.

\section{Clinical findings in patients where microorganisms were detected versus not detected in CSF}

Table 4 shows the clinical signs and symptoms in patients with or without a microorganism detected in CSF. Fever was the most commonly reported symptom, occurring in $81 \%$ of those with a detected microorganism and $74 \%$ of those without. Headache, altered consciousness, seizure, neck stiffness, photophobia and Kernig's and Brudzinski's sign were more common in patients where a microorganism was detected although 


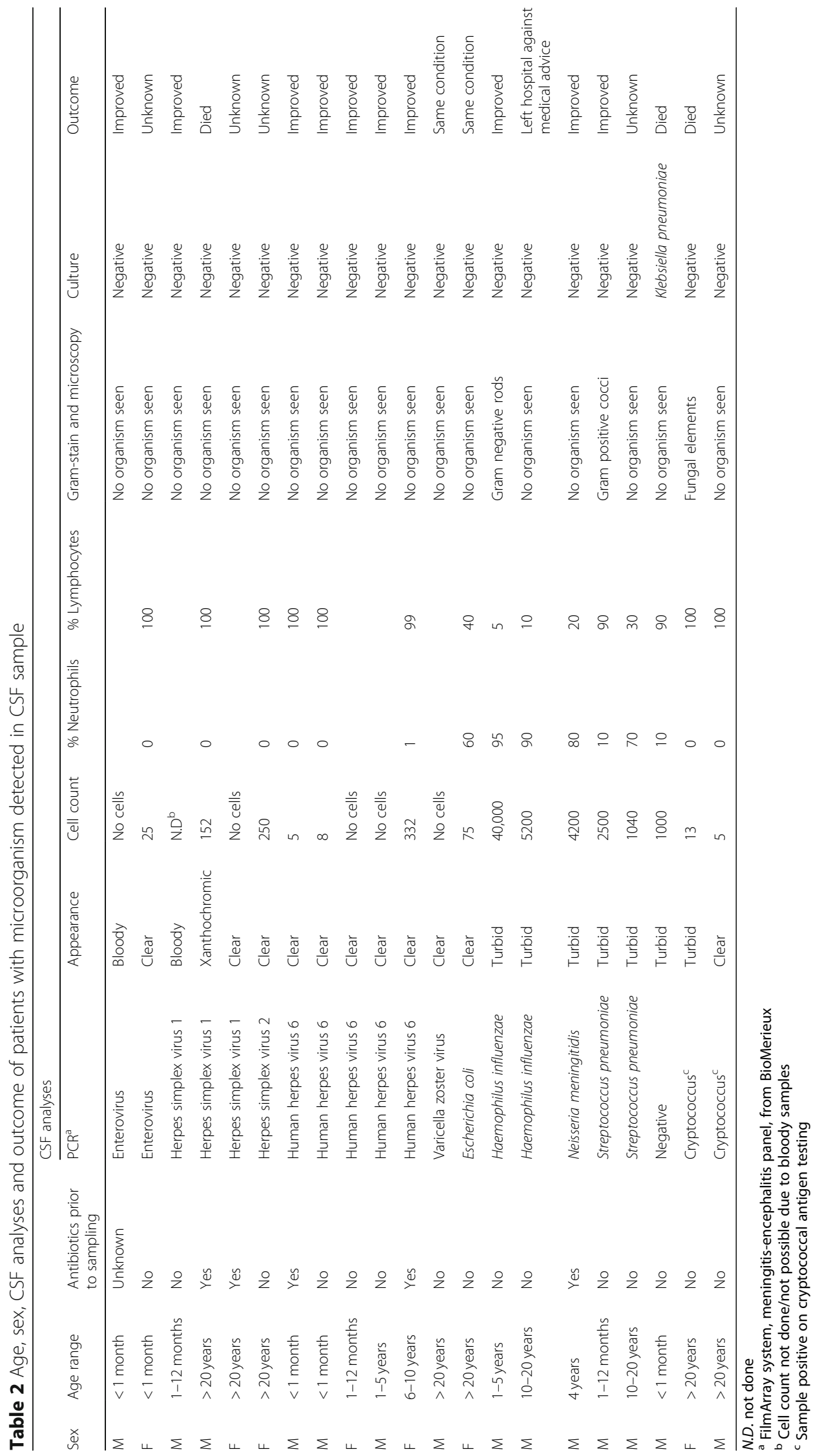


Table 3 Macroscopic appearance and leucocyte count of cerebrospinal fluid (CSF) with respect to confirmed bacterial meningitis

\begin{tabular}{|c|c|c|c|c|}
\hline & \multicolumn{4}{|c|}{ CSF appearance $^{a}$} \\
\hline & \multicolumn{2}{|c|}{ Clear/Xanthochromic } & \multicolumn{2}{|l|}{ Turbid } \\
\hline & Total & Bacterial meningitis $^{\mathrm{b}}$ & $\overline{\text { Total }^{c}}$ & Bacterial meningitis $^{\mathrm{b}}$ \\
\hline \multicolumn{5}{|l|}{ Leukocyte count } \\
\hline$<75$ cells/mm3 & 147 & 0 & $1^{d}$ & 0 \\
\hline$\geq 75$ cells $/ \mathrm{mm} 3$ & 6 & 1 & 8 & 6 \\
\hline
\end{tabular}

${ }^{a}$ In samples containing blood, cell count was not possible. Among 50 bloody samples, none were confirmed to be bacterial meningitis

${ }^{b}$ Confirmed bacterial meningitis defined as bacterial agent detected on PCR or culture

'In one turbid sample, no cell count was performed. No microorganism was detected in this sample

${ }^{C} \mathrm{C}$. neoformans/gattii detected on PCR

these characteristics could not reliably distinguish the patients. This trend was even more prominent when only patients with confirmed bacterial meningitis were considered (data not shown). Bulging fontanel was observed only in three patients, none of whom had microorganism detected in their CSF. No patient was reported to have petechiae.

\section{Antibiotic treatment}

Ninety-seven percent of patients received one or more antibiotic (Table 5). The most commonly used antibiotic was gentamycin, most often in combination with ampicillin, followed by ceftriaxone (data not shown). The majority (90\%) received more than one antibiotic and $39 \%$ received three or more antibiotics. Broad spectrum antibiotics were used in $86 \%$ of cases (data not shown). No difference in the number of antibiotics received, duration of treatment or cumulative days of antibiotics was seen between patients with a microorganism detected and those with negative CSF (Table 5).

\section{Length of stay and outcome}

Data on outcome and duration of hospital stay were available for 161 (74\%) and 172 (79\%) of the patients, respectively (Table 6). The remaining patients were not documented in the registration book or patient files, and data could not be obtained from the ward or in the medical archive.

For patients where the duration of hospital stay was known (74\%), the average length of stay was 9.0 days, and the majority (64\%) of patients had improved upon discharge. There was no significant difference in length of hospital stay for patients with a causative agent identified in CSF (mean 10.0 days) versus those who had a negative CSF sample (mean 9.0 days). The overall case fatality rate was $14 \%$ and there was a trend towards a higher fatality rate in the group that had microorganisms detected in CSF $(18 \%$ versus $12 \%)$, but this was not statistically significant. About $10 \%$ of the patients left the hospital against medical advice and $4 \%$ left in the same condition as when they arrived.

\section{Discussion}

We analysed clinical and microbiological data from prospectively recruited patients with suspected meningitis in a teaching hospital in Ethiopia. The study was performed within the routine clinical and laboratory settings of a hospital that had very limited prior experience with molecular techniques. In addition to the conventional laboratory investigations used in the hospital, a simple and rapid molecular diagnostic system was introduced to enhance laboratory diagnostics during the study period. To the authors' knowledge, this is the first time a definite etiological diagnosis of viral meningitis has been made in patients in a public health facility in Ethiopia.

Virus were the most common etiological agents of meningitis in this study with HHV-6 being the most common, followed by HSV-1 and HSV-2 and enterovirus. Although the etiology is likely to vary between age groups and local epidemiology, this is in agreement with a study from Finland that found enteroviruses, followed by HSV-2 and VZV to be major causes of aseptic meningitis in adults [21] and a study from Brazil that reported enterovirus as the most common cause of meningitis, followed by HSV-1, cytomegalovirus and dengue virus [22].

Early detection of etiologic agents improves the outcome of meningitis [23] and adequate laboratory diagnostics are imperative. Culturing of bacterial and fungal agents takes time and has low sensitivity, as illustrated by the fact that only one of 9 CSF samples with potentially cultivatable organisms was culture positive. The sensitivity of culture is affected by many factors including prior administration of antibiotics, suboptimal culturing conditions and media, and fastidious nature of some of the bacterial agents. Because of this and the unavailability of viral detection, virtually all patients with suspected meningitis in Ethiopia are treated as bacterial meningitis cases and the diagnosis is rarely re-evaluated over the course of the disease. This over-diagnosis of bacterial meningitis inevitably leads to an overuse of antibiotics. Hence, rapid molecular diagnostics can have a major impact in 
Table 4 Signs and symptoms in patients with suspected meningitis with respect to age group and detection of microorganisms in cerebrospinal fluid (CSF)

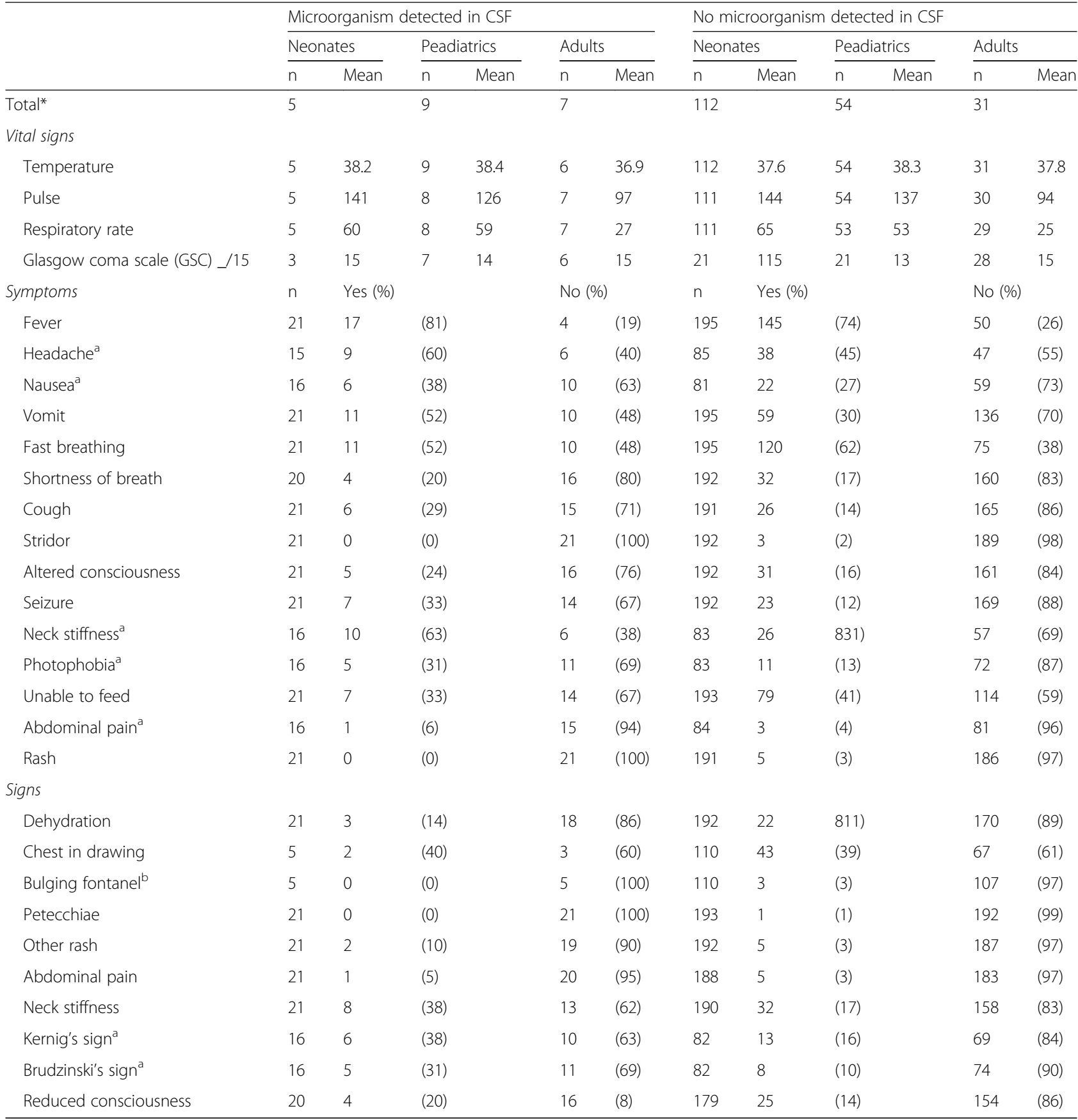

*all patients with suspected meningitis and outcome registered, also patient who had only blood culture and no CSF sample taken

a only applicable for paediatric and adult patients

bonly applicable for neonatal patients

low-income settings by increasing the likelihood of reaching a correct diagnosis and enabling correct patient management. This is crucial not only for the outcome of the individual patient, but also for hospital biosecurity measures, public health decisions and both local and global efforts to reduce and improve antimicrobial usage.
The introduction of syndromic testing of infectious diseases and fully automated multiplexed analyses represents a paradigm shift in microbiological diagnostics [24]. Compared to the conventional laboratory investigations performed at JUSH, that detected a microorganism in CSF of only five patients (two by Gram stain, one by Gram stain and cryptococcal antigen-testing, one by 


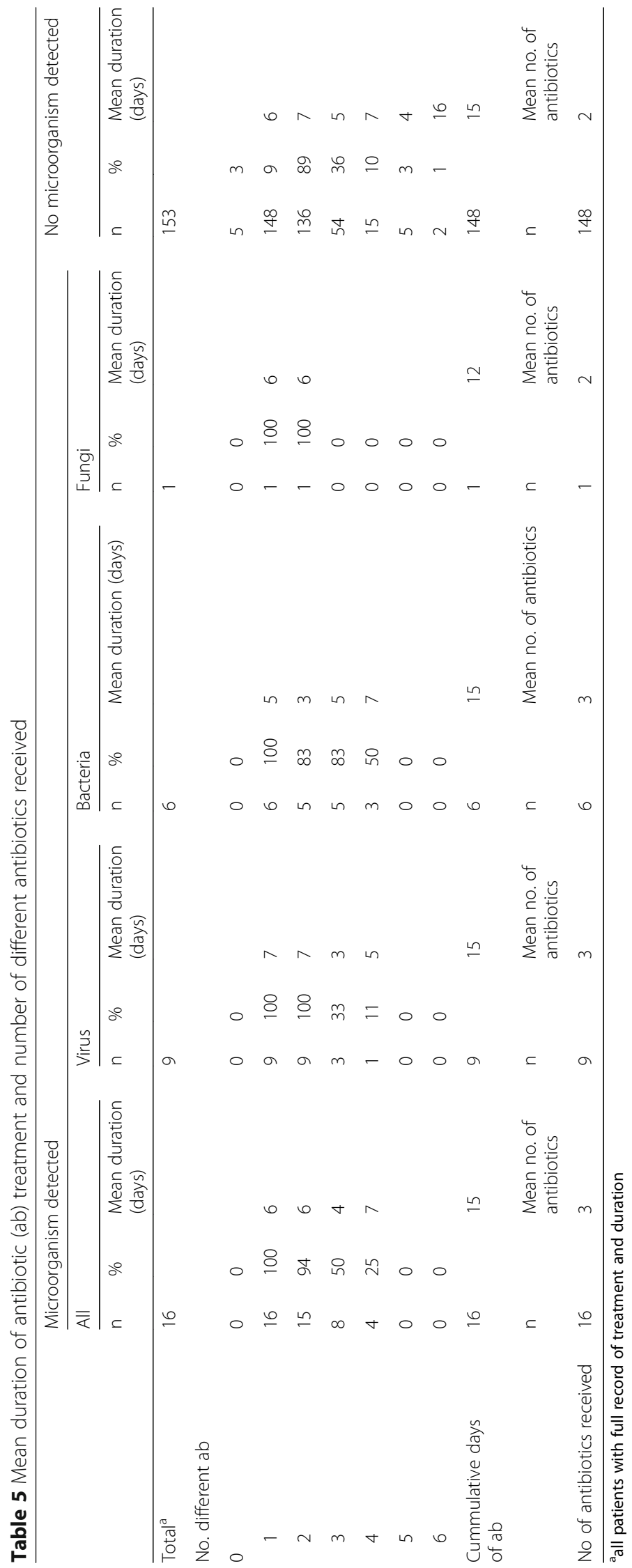


Bårnes et al. BMC Infectious Diseases

(2018) 18:684

Page 11 of 14

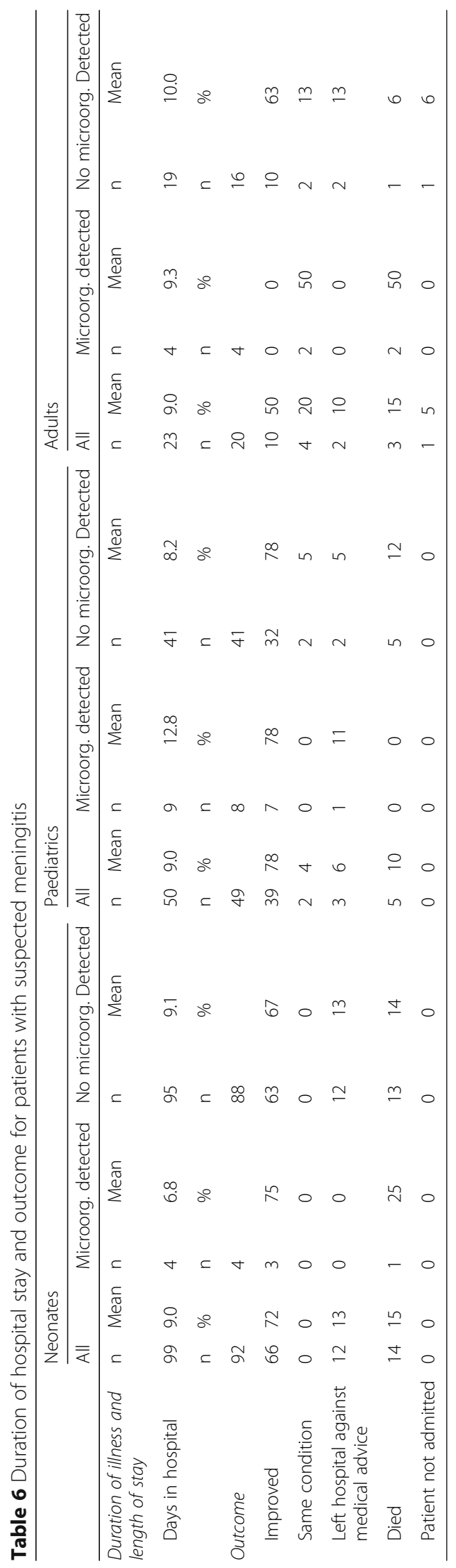


cryptococcal antigen only and one by culture), the FilmArray was able to detect microorganisms in 20 samples using the ME panel. Hence, such systems can improve patient management in settings with limited laboratory facilities. The FilmArray system was very easily implemented into a modestly equipped laboratory where personnel had little prior experience with molecular diagnostics. The laboratory personnel were able to take on the extra task without disrupting their regular working schedule, while the machine operates.

However, there are a number of limitations to the sustained use of such automated systems in low-income countries. In Ethiopia, procurement of the necessary consumables is a complicated and lengthy process. The main obstacle, however, is the cost. Currently, the reagents needed for the analysis of one sample exceed 100 USD. Needless to say this is not sustainable in a public health system that is already financially constrained. On the other hand, a full course of treatment for suspected bacterial meningitis for 10-14 days [25], including only direct expenses for a hospital stay, routine investigations and antibiotic treatment, is likely to amount to more than 100 USD, even in Ethiopia. Another possible limitation of the system is the predefined selection of the pathogens in the panels. The panels were developed for an American market and may not be equally suited for Africa where other pathogens including $M$. tuberculosis and malaria are major causes of infections. It is also important that clinicians have a good understanding of test characteristics, interpretation of results and test limitations. Although lower than for conventional PCR, there is still a potential for sample contamination when using the FilmArray and the assay may detect latent or reactivated viruses [26]. The assays should be used with care and the positivity rates should be monitored.

Molecular diagnostics should not replace conventional methods. Simple investigations like WBC, Gram stain, and glucose and protein measurements provide valuable diagnostic information, helping distinguish between bacterial and non-bacterial meningitis and guiding treatment [7, 23, 27-30]. These simple methods, however, which are defined as routine investigations at JUSH, were not available for most of the study period. No patients in this study had blood/CSF glucose ratio measured, mainly because of challenges in procurement and finances leading to a lack of basic reagents. Nevertheless, the study did confirm that manual cell counts were useful and could be used more actively to influence patient management.

A combination of laboratory diagnostics and clinical examination have been shown to provide the best prediction of bacterial meningitis, with $100 \%$ sensitivity and $52 \%$ specificity [28], but even CSF cell count alone can be a good predictor. A cut-off value of 321 leukocytes/ $\mathrm{mm}^{3}$ showed a sensitivity and specificity for bacterial meningitis of $81 \%$ in a paediatric population in Portugal [27] and all patients with bacterial meningitis in a mixed-age population in Egypt were found to have > 100 leukocytes $/ \mathrm{mm}^{3}$ [29]. In our study, all confirmed cases of bacterial meningitis had $\geq 75$ leukocytes $/ \mathrm{mm}^{3}$. Thus the use of a cut-off value of $\geq 75$ leukocytes $/ \mathrm{mm}^{3}$ and/or a turbid appearance of CSF to indicate bacterial meningitis and the need for antibiotic treatment would have reduced the number of patients treated with antibiotics by almost $75 \%$ in this study.

The high number of patients with no cells or microorganisms detected in the CSF samples indicates that meningitis may be overestimated, especially in children and neonates. Detection of microorganisms in CSF of only $4 \%$ of neonates, might suggest that lumbar puncture, an invasive procedure, is overused. In contrast, the high proportion of positive blood cultures in this study (38\%), particularly in neonates (67\%) suggests that this diagnostic procedure is underused in the hospital.

A limitation of the study is that, despite close follow-up, medical files with treatment and outcome data were not traceable for a considerable proportion (27\%) of the patients. This may have introduced a bias with regards to evaluating outcome. However, the proportion of patients where a microorganism was detected in CSF was the same for the overall study population $(9.9 \%)$ as for the population where outcome was recorded (9.6\%).

No differences were observed in this study with respect to prescription of antibiotics to patients with bacterial/ fungal agents detected in CSF versus those with viral meningitis or no microorganism detected. This could be due to a lack of clinical treatment guidelines, but a contributing factor might have been poor communication of test results to responsible clinicians due to lack of electronic reporting systems, operational hospital telephones and internal post delivery systems. We acknowledge that changes in prescription habits will require long lasting interventions and coordinated stewardships in order to be successful, and that the short duration of the study was a limitation, in this respect. A recent publication from India observed a target specific escalation and evidence based de-escalation of the use of antimicrobials when a syndromic based molecular diagnostic system for meningitis was used over a period of 4 years [31].

The prescription of antibiotics is far higher in Ethiopia than WHO recommendations [32]. There is no formal antimicrobial stewardship program at JUSH, nor restrictions or specific guidelines on antimicrobials use. In Ethiopia, there is no national policy or guidelines on antimicrobials use, neither do most hospitals have their own systems to ensure correct use of antimicrobials [15]. A study from JUSH also showed that the national guidelines did not cover the management of $20 \%$ of 
diagnosed infectious diseases [15]. Ensuring stable access to simple CSF investigations, including protein and glucose measurements, and developing and implementing treatment guidelines for meningitis based on laboratory results might be a first and important step to improve treatment and reduce the misuse of antibiotics.

\section{Conclusions}

In this study we diagnosed viral meningitis in a routine clinical practice for the first time in Ethiopia, and in the study population viral agents were the most common cause of meningitis. A simple, rapid molecular diagnostic system was successfully implemented in a laboratory with little experience in molecular diagnostics, and significantly increased the likelihood of detecting a microorganism in CSF samples. The lack of laboratory methods to effectively distinguish bacterial from nonbacterial causes is an important reason for the overuse of antibiotics in patients with suspected meningitis in Ethiopia. Improved diagnostics together with development of treatment guidelines, based on local epidemiology and laboratory findings, could reduce antibiotic usage and hospitalization without negatively affecting patient outcome in the study hospital.

\section{Additional file}

Additional file 1: Flow chart of cerebrospinal fluid (CRF) sample analysis using the FilmArray system. Description of work flow for sample analysis. (DOCX $29 \mathrm{~kb})$

\section{Abbreviations}

BCID: Blood Culture Identification; CSF: Cerebrospinal fluid; JUSH: Jimma University Specialized Hospital; ME: Meningitis/encephalitis; PMN: Plymorphonuclear leukocytes cells; USD: US Dollar; WBC: White blood cells

\section{Acknowledgments}

We thank all the study participants and the clinical staff at Jimma University Specialized Hospital, particularly in the paediatric and internal medicine wards. We are very grateful to Dawit Abera, Amanuel Teferi, Wossen Abay and Sara Jemal for performing the PCR and the routine analysis of CSF samples and blood cultures. We are also grateful to Mulualem Tadesse, Zegeye Bonsa, Nefiza Negash and Getu Belay for running the geneXpert analysis of CSF samples.

\section{Funding}

This project was supported by the Research Council of Norway grant 220829 to DAC. The funding body has not been involved in the design of the study and collection, analysis, and interpretation of data or in writing the manuscript.

\section{Availability of data and materials}

Data used for this study is available on request.

\section{Authors' contributions}

GKB, EKG, HJJ, MB, SLF, AA and DAC participated in the study design. GKB, $E K G, M B$ and GT were responsible for recruitment and sampling. GKB, GT, $A A$ and GA were responsible for the laboratory analyses. GKB, EKG, HJJ and SLF analysed the data. GKB, HJJ and SLF drafted the manuscript. All the authors have contributed to the manuscript and approved the final version.

\section{Competing interest}

The authors declare that they have no competing interests.

\section{Ethics approval and consent to participate}

The study obtained ethical approval from the Regional Committees for Medical and Health Research Ethics in Norway and the Jimma University Institutional Review Board of the Institute of Health. Study information was given in the local language and written informed consent was obtained from all participants, or by parents or adult care takers in the case of children under the age of 16

\section{Consent for publication}

Not applicable. This manuscript does not contain any individual persons' data.

\section{Publisher's Note}

Springer Nature remains neutral with regard to jurisdictional claims in published maps and institutional affiliations.

\section{Author details}

${ }^{1}$ Division for Infection Control and Environmental Health, Norwegian Institute of Public Health, Oslo, Norway. ${ }^{2}$ Faculty of Medicine, University of Oslo, Oslo, Norway. ${ }^{3}$ Institute of Health, Jimma University, Jimma, Ethiopia. ${ }^{4}$ Norwegian Veterinary Institute, Oslo, Norway. ${ }^{5}$ Mycobacteriology Research Center, Jimma University, Jimma, Ethiopia.

Received: 12 February 2018 Accepted: 4 December 2018

Published online: 20 December 2018

\section{References}

1. Institute of Health Metrics and Evaluation. Leading causes of premature death (YLLs) in 2015 and Leading causes of DALYs in 2015. Website: healthdata.org/ethiopia. Accessed 10 July 2017.

2. Colombini A, Bationo F, Zongo S, Ouattara F, Badolo O, Jaillard P, et al. Costs for households and community perception of meningitis epidemics in Burkina Faso. Clin Infect Dis. 2009:49:1520-5.

3. Barquet N, Domingo P, Cayla JA, Gonzalez J, Rodrigo C, Fernandez-Viladrich $P$, et al. Prognostic factors in meningococcal disease. Development of a bedside predictive model and scoring system. JAMA. 1997;278:491-6.

4. Shah SS, Aronson PL, Mohamad Z, Lorch SA. Delayed acyclovir therapy and death among neonates with herpes simplex virus infection. Pediatrics. 2011; 128:1153-60.

5. Tunkel AR, Hartman BJ, Kaplan SL, Kaufman BA, Roos KL, Scheld WM, et al. Practice guidelines for the management of bacterial meningitis. Clin Infect Dis. 2004;39:1267-84.

6. Biondi EA, Byington CL. Evaluation and management of febrile, wellappearing young infants. Infect Dis Clin N Am. 2015;29:575-85.

7. Dubos F, Lamotte B, Bibi-Triki F, Moulin F, Raymond J, Gendrel D, et al. Clinical decision rules to distinguish between bacterial and aseptic meningitis. Arch Dis Child. 2006;91:647-50.

8. Neuman MI, Tolford S, Harper MB. Test characteristics and interpretation of cerebrospinal fluid gram stain in children. Pediatr Infect Dis J. 2008;27:309-13.

9. Hornik CP, Benjamin DK, Becker KC, Benjamin DK, Li J, Clark RH, et al. use of the complete blood cell count in early-onset neonatal sepsis. Pediatr Infect Dis J. 2012;31:799-802

10. Jaffe DM, Fleisher GR. Temperature and total white blood cell count as indicators of bacteremia. Pediatrics. 1991;87:670-4.

11. The World Bank. Overview Ethiopia. Website: worldbank.org/en/country/ ethiopia/overview\#1. Accessed: 01112017.

12. Gudina EK, Tesfaye M, Adane A, Lemma K, Shibiru T, Pfister HW, Klein M. Challenges of bacterial meningitis case management in low income settings: an experience from Ethiopia. Tropical Med Int Health. 2016;21:870-8.

13. Thomas KE, Hasbun R, Jekel J, Quagliarello VJ. The diagnostic accuracy of Kernig's sign, Brudzinski's sign, and nuchal rigidity in adults with suspected meningitis. Clin Infect Dis. 2002;35:46-52.

14. Oostenbrink R, Moons KG, Theunissen CC, Derksen-Lubsen G, Grobbee DE, Moll HA. Signs of meningeal irritation at the emergency department: how often bacterial meningitis? Pediatr Emerg Care. 2001;17:161-4.

15. Yadesa TM, Gudina EK, Angamo MT. Antimicrobial use-related problems and predictors among hospitalized medical in-patients in Southwest Ethiopia: prospective observational study. PLoS One. 2015;10:e138385.

16. Moges F, Endris M, Mulu A, Tessema B, Belyhun Y, Shiferaw Y, et al. The growing challenges of antibacterial drug resistance in Ethiopia. J Glob Antimicrob Resist. 2014;2:148-54. 
17. Cheesbrough M. District laboratory practice in tropical countries. 2nd ed Cambridge: Cambridge University press; 2006.

18. Leber AL, Everhart K, Balada-Llasat JM, Cullison J, Daly J, Holt S, et al. Multicenter evaluation of Biofire FilmArray meningitis/encephalitis panel for detection of bacteria, viruses, and yeast in cerebrospinal fluid specimens. J Clin Microbiol. 2016:54:2251-61.

19. Helb D, Jones M, Story E, Boehme C, Wallace E, Ho K, et al. Rapid detection of Mycobacterium tuberculosis and rifampin resistance by use of on-demand near-patient technology. J Clin Microbiol. 2010;48:229-37.

20. Blaschke AJ, Heyrend C, Byington CL, Fisher MA, Barker E, Garrone NF, et al. Rapid identification of pathogens from positive blood cultures by multiplex polymerase chain reaction using the FilmArray system. Diagn Microbiol Infect Disease. 2012;74:349-55.

21. Kupila L, Vuorinen T, Vainionpaa R, Hukkanen V, Marttila RJ, Kotilainen P. Etiology of aseptic meningitis and encephalitis in an adult population. Neurology. 2006;66:75-80.

22. Soares CN, Cabral-Castro MJ, Peralta JM, de Freitas MR, Zalis M, PuccioniSohler M. Review of the etiologies of viral meningitis and encephalitis in a dengue endemic region. J Neurol Sci. 2011;303:75-9.

23. Bahr NC, Boulware DR. Methods of rapid diagnosis for the etiology of meningitis in adults. Biomark Med. 2014;8:1085-103.

24. Abbott AN, Fang FC. Clinical impact of multiplex syndromic panels in the diagnosis of bloodstream, gastrointestinal, respiratory. and central nervous system infections Clin Microbiol Newsl. 2017;39:133-42.

25. Food, Medicine and Healthcare Administration and Control Authority of Ethiopia (FMHACA). Standard treatment guideline for general hospital. In: $3^{\text {rd }}$ edition; 2014

26. Hanson KE. The first fully automated molecular diagnostic panel for meningitis and encephalitis: how well does it perform, and when should it be used? J Clin Microbiol. 2016;54:2222-4.

27. Agueda S, Campos T, Maia A. Prediction of bacterial meningitis based on cerebrospinal fluid pleocytosis in children. Braz J Infect Dis. 2013;17:401-4.

28. Dubos F, Korczowski B, Aygun DA, Martinot A, Prat C, Galetto-Lacour A, et al. Distinguishing between bacterial and aseptic meningitis in children: European comparison of two clinical decision rules. Arch Dis Child. 2010;95: 963-7.

29. Fouad R, Khairy M, Fathalah W, Gad T, El-Kholy B, Yosry A. Role of clinical presentations and routine CSF analysis in the rapid diagnosis of acute bacterial meningitis in cases of negative gram stained smears. J Trop Med. 2014:213762.

30. Spanos A, Harrell FE Jr, Durack DT. Differential diagnosis of acute meningitis An analysis of the predictive value of initial observations. JAMA. 1989;262: 2700-7.

31. Javali M, Acharya P, Mehta A, John AA, Mahale R, Srinivasa R. Use of multiplex PCR based molecular diagnostics in diagnosis of suspected CNS infections in tertiary care setting-a retrospective study. Clin Neurol Neurosurg. 2017;161:110-6.

32. Kebede HK, Gesesew HA, Woldehaimanot TE, Goro KK. Antimicrobial use in paediatric patients in a teaching hospital in Ethiopia. PLoS One. 2017;12: e0173290.

Ready to submit your research? Choose BMC and benefit from:

- fast, convenient online submission

- thorough peer review by experienced researchers in your field

- rapid publication on acceptance

- support for research data, including large and complex data types

- gold Open Access which fosters wider collaboration and increased citations

- maximum visibility for your research: over $100 \mathrm{M}$ website views per year

At $\mathrm{BMC}$, research is always in progress.

Learn more biomedcentral.com/submissions 